\title{
Partial Feedback Based Orthogonal Space-Time Block Coding With Flexible Feedback Bits"
}

\author{
Lei Wang, Zhigang Chen \\ School of Electronics and Information Engineering, Xi'an Jiaotong University, Xi'an, China \\ Email: lei.wang@mail.xjtu.edu.cn,zgchen@mail.xjtu.edu.cn
}

Received June, 2013

\begin{abstract}
The conventional orthogonal space-time block code (OSTBC) with limited feedback has fixed $p-1$ feedback bits for the specific $n_{T} p$ transmit antennas. A new partial feedback based OSTBC which provides flexible feedback bits is proposed in this paper. The proposed scheme inherits the properties of having a simple decoder and the full diversity of OSTBC, moreover, preserves full data rate. Simulation results show that for $n_{T} p$ transmit antennas, the proposed scheme has the similar performance with the conventional one by using $p-1$ feedback bits, whereas has the better performance with more feedback bits.
\end{abstract}

Keywords: MIMO; Transmit Diversity; Space-time Block Coding; Partial Feedback

\section{Introduction}

Orthogonal space-time block coding (OSTBC) is a simple and effective transmission paradigm for MIMO system, due to achieving full diversity with low complexity [1]. One of the most effective OSTBC schemes is the Alamouti code [2] for two transmit antennas, which has been adopted as the open-loop transmit diversity scheme by current 3GPP standards. However, the Alamouti code is the only rate-one OSTBC scheme [3]. With higher number of transmit antennas, the OSTBC for complex constellations will suffer the rate loss.

Focusing on this drawback, the open-loop solutions have been presented, such as the quasi-OSTBC (QOSTBC) [4] with rate one for four transmit antennas, and other STBC schemes $[5,6]$ with full rate and full diversity. Alternatively, the close-loop solutions have also been designed to improve the performance of OSTBC by exploiting limited channel information feedback at the transmitter. In this paper, we focus on the close-loop scheme.

Based the group-coherent code, the $p-1$ bits feedback based OSTBC for $n_{T} p$ transmit antennas has been constructed in [7], and generalized to an arbitrary number of receive antennas in [8]. The partial feedback based

${ }^{*}$ This work was supported by the National Natural Science Foundation of China under Grant 60902045, the International Cooperation Projects of China-Finland under Grant 2010DFB10570, and the National Science \& Technology Major Projects of China under Grant 2010ZX03003-004. schemes in $[7,8]$ exhibit a higher diversity order while preserving low decoding complexity. However, these schemes for $n_{T} p$ transmit antennas require a fixed number of $p-1$ bits feedback. That is to say, for such scheme, improving the performance by increasing the feedback bits implies that the number of transmit antennas $n_{T} p$ must be increased at the same time. Therefore, the scheme is inflexible in compromising the performance and the feedback overhead.

In this paper, by multiplying a well-designed feedback vector to each signal to be transmitted from each antenna, we propose a novel partial feedback based OSTBC scheme with flexible feedback bits. In this scheme, the OSTBC can be straightly extended to more than two antennas. Importantly, we can show that the proposed scheme preserves the simple decoding structure of OSTBC, full diversity and full data rate.

Notations: Throughout this paper, $(\cdot)^{T}$ and $(\cdot)^{H}$ represent "transpose" and "Hermition", respectively. $\operatorname{Re}(a)$ denotes the real part of a complex $a$, and $j=\sqrt{-1}$.

\section{Proposed Code Construction and System Model}

Consider a MIMO system with $n_{T} p$ transmit and $n_{R}$ receive antennas. Assuming we have an OSTBC $\mathbf{G}_{n_{T}}$ for $n_{T}$ transmit antennas, and $\mathbf{G}_{n_{T}}$ can be denoted as $\mathbf{G}_{n_{T}}=\left[\begin{array}{llll}\mathbf{c}_{1} & \mathbf{c}_{2} & \cdots & \mathbf{c}_{n_{T}}\end{array}\right]$, where $\mathbf{c}_{m}$ is the $T \times 1$ sig- 
nal to be transmitted from the $m$ th antenna for $m=1, \cdots, n_{T}$. Then a code to be transmitted from $n_{T} p$ antennas, where $p \geq 2$ is an integer, may be constructed as

$$
\mathbf{G}_{n_{T} p}^{l}=\sum_{m=1}^{n_{T}} \mathbf{c}_{m} \boldsymbol{\beta}_{m}^{l}
$$

where $\boldsymbol{\beta}_{m}^{l}$ is the $1 \times n_{T} p$ feedback vector for the $m$ th antenna, which is defined as $\boldsymbol{\beta}_{m}^{l}=\boldsymbol{\varphi}_{m} \otimes \mathbf{b}^{l}$, where $\otimes$ denotes the Kronecker product, $\boldsymbol{\varphi}_{m}$ is the $m$ th row of the identity matrix $\mathbf{I}_{n_{T}}$, and $1 \times p$ vector $\mathbf{b}^{l}$ is given by

$$
\mathbf{b}^{l}:=\left[\begin{array}{llll}
1 & e^{j(2 \pi / Q) b_{1}} & \cdots & e^{j(2 \pi / Q) b_{p-1}}
\end{array}\right]
$$

where $b_{1}, \cdots, b_{p-1} \in A=\{0,1, \cdots, Q-1\}$. For the feedback vector at the $m$ th antenna, it contains a subset of all possible $Q^{p-1}$ feedback vectors $\boldsymbol{\beta}_{m}^{l}$, i.e., $l=1,2, \cdots, Q^{p-1}$.

With the transmission of $T \times n_{T} p$ code matrix $\mathbf{G}_{n_{T} p}^{l}$, the $T \times n_{R}$ receive signal $\mathbf{Y}=\left[\mathbf{y}_{1}, \cdots, \mathbf{y}_{n_{R}}\right]$ can be written as

$$
\mathbf{Y}=\mathbf{G}_{n_{T} p}^{l} \mathbf{H}+\mathbf{N}
$$

where $\mathbf{H}=\left[\mathbf{h}_{1}, \cdots, \mathbf{h}_{n_{R}}\right]$ is the $n_{T} p \times n_{R}$ channel matrix, and $\mathbf{N}=\left[\mathbf{n}_{1}, \cdots, \mathbf{n}_{n_{R}}\right]$ is the $T \times n_{R}$ complex Gaussian noise matrix. The entries of $\mathbf{H}$ and $\mathbf{N}$ are independent samples of a zero-mean complex Gaussian random variable with variance 1 and $n_{T} p / \rho$ respectively, where $\rho$ is the average signal-to-noise ratio (SNR) at each receive antenna.

\section{Linear Decoder at the Receiver}

The received signal at $i$ th receive antenna can be rewritten as

$$
\begin{aligned}
\mathbf{y}_{i} & =\mathbf{G}_{n_{T} p}^{l} \mathbf{h}_{i}+\mathbf{n}_{i}=\mathbf{G}_{n_{T}} \mathbf{B}^{l} \mathbf{h}_{i}+\mathbf{n}_{i} \\
& =\mathbf{G}_{n_{T}} \tilde{\mathbf{h}}_{i}^{l}+\mathbf{n}_{i}
\end{aligned}
$$

where the $n_{T} \times n_{T} p$ matrix $\mathbf{B}^{l}$ is composed of $n_{T}$ feedback vectors, and can be expressed in a stacked form given by

$$
\mathbf{B}^{l}=\left[\begin{array}{lll}
\left(\boldsymbol{\beta}_{1}^{l}\right)^{T} & \cdots & \left(\boldsymbol{\beta}_{n_{T}}^{l}\right)^{T}
\end{array}\right]^{T} .
$$

We divide $n_{T} p \times 1$ channel vector $\mathbf{h}_{i}$ into $n_{T}$ segments in the following way

$$
\mathbf{h}_{i}=[\underbrace{h_{i, 1}, \cdots, h_{i, p}}_{:=\mathbf{g}_{i 1}^{T}}, \cdots \cdots, \underbrace{h_{i,\left(n_{T}-1\right) p+1}, \cdots, h_{i, p n_{T}}}_{:=\mathbf{g}_{i n_{T}}^{T}}]^{T}
$$

where each segment can be denoted as $\mathbf{g}_{i k}\left(k=1, \cdots, n_{T}\right)$ with dimension $p \times 1$. Then the equivalent channel vec- tor $\tilde{\mathbf{h}}_{i}^{l}=\mathbf{B}^{l} \mathbf{h}_{i}$ in (4) has the form of

$$
\begin{aligned}
\tilde{\mathbf{h}}_{i}^{l} & =\left[\begin{array}{llll}
\left(\boldsymbol{\varphi}_{1} \otimes \mathbf{b}^{l}\right) \mathbf{h}_{i} & \cdots & \left(\boldsymbol{\varphi}_{n_{T}} \otimes \mathbf{b}^{l}\right) \mathbf{h}_{i}
\end{array}\right]^{T} \\
& =\left[\begin{array}{llll}
\mathbf{b}^{l} \mathbf{g}_{i 1} & \mathbf{b}^{l} \mathbf{g}_{i 2} & \cdots & \mathbf{b}^{l} \mathbf{g}_{i n_{T}}
\end{array}\right]^{T}
\end{aligned}
$$

For convenience, we will use the Alamouti code as the basic OSTBC matrix $\mathbf{G}_{n_{T}}$ in the rest of this paper, and the results can be straightly extended to other OSTBC. For the received signal in (4), After performing the conjugate operation to the second entry of $\mathbf{y}_{i}$, the received signal $\mathbf{y}_{i}$ can be equivalently expressed as

$$
\tilde{\mathbf{y}}_{i}=\mathbf{R}_{i}^{l} \mathbf{x}+\tilde{\mathbf{n}}_{i}
$$

where $\mathbf{R}_{i}^{l}$ is the equivalent channel matrix corresponding to the entries of $\tilde{\mathbf{h}}_{i}^{l}$ and their conjugates, and $\mathbf{x}=\left[\begin{array}{ll}s_{1} & s_{2}\end{array}\right]^{T}$ has a pair of symbols in the Alamouti code. Denote the $k$ th entry of $\tilde{\mathbf{h}}_{i}^{l}$ as $\tilde{\mathbf{h}}_{i}^{l}(k)$, and according to the linearity of the OSTBC [9], the equivalent channel matrix $\mathbf{R}_{i}^{l}$ has the form of

$$
\mathbf{R}_{i}^{l}=\sum_{k=1}^{2}\left[\mathbf{C}_{k} \tilde{\mathbf{h}}_{i}^{l}(k)+\mathbf{D}_{k}\left(\tilde{\mathbf{h}}_{i}^{l}(k)\right)^{*}\right]
$$

where the matrices $\mathbf{C}_{k}$ and $\mathbf{D}_{k}$ specifying the Alamouti code are defined in [9]. Since matched filtering is the first step in the detection process, left-multiplying $\tilde{\mathbf{y}}_{i}$ by $\left(\mathbf{R}_{i}^{l}\right)^{H}$ will yield

$$
\mathbf{r}_{i}=\left(\mathbf{R}_{i}^{l}\right)^{H} \tilde{\mathbf{y}}_{i}=\mathbf{M}_{i}^{l} \mathbf{x}+\left(\mathbf{R}_{i}^{l}\right)^{H} \tilde{\mathbf{n}}_{i}
$$

where $\mathbf{M}_{i}^{l}=\left(\mathbf{R}_{i}^{l}\right)^{H}\left(\mathbf{R}_{i}^{l}\right)$. Due to the properties of $\mathbf{C}_{k}$ and $\mathbf{D}_{k}$ for the Alamouti code, we get

$$
\begin{aligned}
\mathbf{M}_{i}^{l} & =\left(\left|\tilde{\mathbf{h}}_{i}^{l}(1)\right|^{2}+\left|\tilde{\mathbf{h}}_{i}^{l}(2)\right|^{2}\right)\left(\mathbf{C}_{1}-\mathbf{D}_{1}\right) \\
& =\left\|\tilde{\mathbf{h}}_{i}^{l}\right\|^{2} \mathbf{I}_{2}=\gamma_{i}^{l} \mathbf{I}_{2}
\end{aligned}
$$

where $\gamma_{i}^{l}$ denotes the equivalent channel gain for receive antenna $i$. It is clear that $\mathbf{M}_{i}^{l}$ is a diagonal matrix, therefore, the simple decoder of OSTBC can be straightly applied for (7), thus $s_{1}$ and $s_{2}$ can be decoded independently.

\section{Feedback Bits Selection and Properties}

In this section, we will discuss the feedback bits selection criterion and the key properties of the proposed scheme.

\subsection{Feedback Bits Selection}

At the $i$ th receive antenna, $\gamma_{i}^{l}$ can be expressed in the following quadratic form

$$
\gamma_{i}^{l}=\left\|\tilde{\mathbf{h}}_{i}^{l}\right\|^{2}=\left|\mathbf{b}^{l} \mathbf{g}_{i 1}\right|^{2}+\left|\mathbf{b}^{l} \mathbf{g}_{i 2}\right|^{2}=\sum_{k=1}^{2} \mathbf{g}_{i k}^{H} \mathbf{A}^{l} \mathbf{g}_{i k}
$$


where

$$
\mathbf{A}^{l}:=\left(\mathbf{b}^{l}\right)^{H} \mathbf{b}^{l}=\left[\begin{array}{cccc}
1 & \alpha^{b_{1}} & \cdots & \alpha^{b_{p-1}} \\
\alpha^{-b_{1}} & 1 & \cdots & \alpha^{\left(b_{p-1}-b_{1}\right)} \\
\vdots & \vdots & \ddots & \vdots \\
\alpha^{-b_{p-1}} & \alpha^{-\left(b_{p-1}-b_{1}\right)} & \cdots & 1
\end{array}\right],
$$

and $\alpha=e^{j(2 \pi / Q)}$.

For all the $n_{R}$ receive antennas, then the total channel gain is given by

$$
\gamma^{l}=\sum_{i=1}^{n_{R}} \gamma_{i}^{l}=\sum_{i=1}^{n_{R}} \sum_{k=1}^{2} \mathbf{g}_{i k}^{H} \mathbf{A}^{l} \mathbf{g}_{i k}, l=1,2, \cdots, Q^{p-1}
$$

It is clear that in order to improve the system performance, we must feedback the specific $l$ with $(p-1) \log Q$ bits, which provides the largest $l \gamma^{l}$. Denote the $(m, n)$ entry of $\mathbf{A}^{l}$ as $\mathbf{A}_{m n}^{l}$, thus $\mathbf{A}_{m m}^{l}=1$, and $\mathbf{A}_{m n}^{l}=\alpha^{\left(b_{n-1}-b_{m-1}\right)}$, where $b_{0}=0$ is preset. Moreover, it is easy to verify that $\mathbf{A}_{m n}^{l}=\left(\mathbf{A}_{n m}^{l}\right)$. Then the quadratic form in (11) can be represented as

$$
\begin{aligned}
\mathbf{g}_{i k}^{H} \mathbf{A}^{l} \mathbf{g}_{i k} & =\sum_{m=1}^{p} \sum_{n=1}^{p} \mathbf{A}_{m n}^{l} \mathbf{g}_{i k}^{*}(m) \mathbf{g}_{i k}(n) \\
& =\left|\mathbf{g}_{i k}\right|^{2}+\sum_{m=2}^{p} \sum_{n=1, n<m}^{p-1} 2 \operatorname{Re}\left[\mathbf{g}_{i k}^{*}(m) \mathbf{g}_{i k}(n) \mathbf{A}_{m n}^{l}\right]
\end{aligned}
$$

where $\mathbf{g}_{i k}(n)$ denotes the $n$th element in $\mathbf{g}_{i k}$, and

$$
\mathbf{g}_{i k}(n)=h_{i,(k-1) p+n} .
$$

Substituting (13) in (11) and $\gamma^{l}=\sum_{i=1}^{n_{R}} \gamma_{i}^{l}$ leads to

$$
\gamma^{l}=\|\mathbf{H}\|_{F}^{2}+\underbrace{\sum_{i=1}^{n_{R}} \sum_{k=1}^{2} \sum_{m=2}^{p} \sum_{n=1, n<m}^{p-1} 2 \operatorname{Re}\left[h_{i,(k-1) p+m}^{*} h_{i,(k-1) p+n} \mathbf{A}_{m n}^{l}\right]}_{:=\zeta}
$$

Thus, the $(p-1) \log Q$ feedback bits will be selected as

$$
l_{\text {opt }}=\arg \max _{l} \zeta, \quad l=1,2, \cdots, Q^{p-1}
$$

In this way, we can choose the optimal feedback vector $\mathbf{b}^{l}$, further construct $\boldsymbol{\beta}_{m}^{l}=\boldsymbol{\varphi}_{m} \otimes \mathbf{b}^{l}$ for the $m$ th transmit antenna.

\subsection{Diversity Analysis}

The key property of the proposed partial feedback based OSTBC scheme is proved in the following.

Property 1: The partial feedback based OSTBC $\mathbf{G}_{n_{T} p}^{l}$ in (1) can achieve full diversity.

Proof: For simplicity, we denote $L=Q^{p-1}$. Selecting the optimal $l_{\text {opt }}$ will provide the largest channel gain $\max \left(\gamma^{1}, \cdots, \gamma^{L}\right)$, which can be lower bounded by

$$
\begin{aligned}
& \max \left(\gamma^{1}, \cdots, \gamma^{L}\right) \geq \bar{\gamma}=\frac{1}{L} \sum_{l=1}^{L} \gamma^{l} \\
& =\frac{1}{L} \sum_{l=1}^{L} \sum_{i=1}^{n_{R}} \sum_{k=1}^{2} \mathbf{g}_{i k}^{H} \mathbf{A}^{l} \mathbf{g}_{i k}=\frac{1}{L} \sum_{i=1}^{n_{R}} \sum_{k=1}^{2} \mathbf{g}_{i k}^{H}\left(\sum_{l=1}^{L} \mathbf{A}^{l}\right) \mathbf{g}_{i k}
\end{aligned}
$$

For the summed matrix $\sum_{l=1}^{L} \mathbf{A}^{l}$, it is clear that its diagonal elements equal to $L$, and its non-diagonal elements have the form of

$$
\sum_{l=1, m \neq n}^{L} \mathbf{A}_{m n}^{l}=\sum_{\substack{0 \leq b_{n-1}, b_{m-1} \leq Q-1, m \neq n}} \alpha^{\left(b_{n-1}-b_{m-1}\right)}
$$

Let $k=b_{n-1}-b_{m-1}$, since $0 \leq k \leq Q-1$, (17) is reduced to

$$
\sum_{l=1, m \neq n}^{L} \mathbf{A}_{m n}^{l}=\sum_{k=0}^{Q-1} \alpha^{k}=0
$$

Therefore, we can obtain $\sum_{l=1}^{L} \mathbf{A}^{l}=L \mathbf{I}_{p}$, which can be substituted into (16) and yields

$$
\begin{aligned}
\bar{\gamma} & =\frac{1}{L} \sum_{l=1}^{L} \gamma^{l}=\sum_{i=1}^{n_{R}} \sum_{k=1}^{2} \mathbf{g}_{i k}^{H} \mathbf{g}_{i k}=\sum_{i=1}^{n_{R}}\left(\left\|\mathbf{g}_{i 1}\right\|^{2}+\left\|\mathbf{g}_{i 2}\right\|^{2}\right) \\
& =\sum_{i=1}^{n_{R}}\left\|\mathbf{h}_{i}\right\|^{2}=\|\mathbf{H}\|_{F}^{2}
\end{aligned}
$$

Since the lower bound of the channel gain provides full diversity of $n_{T} p n_{R}$, the proposed scheme can certainly guarantee the full diversity.

\subsection{Configuration of Flexible Feedback Bits}

Furthermore, the proposed scheme $\mathbf{G}_{n_{T} p}^{l}$ has the flexible feedback bits. For a specific $p, \mathbf{G}_{n_{T} p}^{T}$ has the feedback bits of $(p-1) \log Q$. However, for the number that not equal to $(p-1) \log Q$, we can rewrite the vector $\mathbf{b}^{l}$ in (2) as $\mathbf{b}^{l}=\left[\begin{array}{llll}1 & e^{j\left(2 \pi / Q_{1}\right) b_{1}} & \cdots & e^{j\left(2 \pi / Q_{p-1}\right) b_{p-1}}\end{array}\right]$, thus the number of feedback bits is $\log \prod_{i=1}^{p-1} Q_{i}$. For example, for $n_{T}=2$ and $p=4$, the number of feedback bits are 3 and 6 in the case of $Q=2$, and $Q=4$, respectively. If we set $Q_{1}=Q_{2}=2$, and $Q_{3}=4$ in $\mathbf{b}^{l}$, then the number of feedback bits is 4 , and if we set $Q_{1}=2$, and $Q_{2}=Q_{3}=4$ in $\mathbf{b}^{l}$, then the number of feedback bits is 5 , and so on.

\subsection{BER Analysis}

Assuming the power of each symbol in $\mathbf{x}=\left[\begin{array}{ll}s_{1} & S_{2}\end{array}\right]^{T}$ is normalized to unity, i.e., $E\left\{\left|s_{i}\right|^{2}\right\}=1$ for $i=1,2$, we can obtain the average SNR per bit has the form of

$$
\gamma_{b}=\frac{\rho}{2 n_{T} p} \max \left(\gamma^{1}, \cdots, \gamma^{L}\right) .
$$


Furthermore, assuming QPSK modulation and maximum likelihood (ML) decoding are used in the considered system, the conditional BER is given by

$$
p_{b}\left(e \mid \gamma_{b}\right)=Q\left(\sqrt{2 \gamma_{b}}\right)
$$

By using (16), the upper bound of the conditional BER can be formulated as

$$
p_{b}\left(e \mid \gamma_{b}\right) \leq Q\left(\sqrt{\frac{\rho}{n_{T} p} \bar{\gamma}}\right)=p_{b}^{u}(e \mid \bar{\gamma})
$$

Using the technique of Moment Generating Function (MGF)[10], the average BER can be expressed as

$$
p_{b}^{u}=\frac{1}{\pi} \int_{0}^{\pi / 2} M_{\eta}\left(-\frac{1}{2 \sin ^{2} \theta}\right) d \theta
$$

where $\eta=\frac{\rho}{n_{T} p} \bar{\gamma}$, and $M_{\eta}(s)=\left(1-\frac{\rho}{n_{T} p} s\right)^{-n_{T} n_{R} p}$

is the MGF of $\eta$. The average BER can be further expressed as

$$
p_{b}^{u}=\frac{1}{\pi} \int_{0}^{\pi / 2}\left(\frac{\sin ^{2} \theta}{\sin ^{2} \theta+\rho /\left(2 n_{T} p\right)}\right)^{n_{T} n_{R} p} d \theta
$$

Using the result of (5A.4) in [10], this definite integrals has the closed-form of

$$
p_{b}^{u}=\left(\frac{1-\mu}{2}\right)^{n_{T} n_{R} p} \sum_{k=0}^{n_{T} n_{R} p-1}\left(\begin{array}{c}
n_{T} n_{R} p-1+k \\
k
\end{array}\right)\left(\frac{1+\mu}{2}\right)^{k}
$$

where $\mu=\sqrt{\frac{\rho}{2 n_{T} p+\rho}}$.

\section{Simulation Results}

In all simulations, we consider QPSK symbols in Alamouti code, and a single receive antenna with $n_{R}=1$, where the channels are assumed to be independent and identically distributed (i.i.d.) quasi-static Rayleigh flatfading channels. In Figure 1, we plot the bit error rate (BER) performance of the generalized partial feedback based OSTBC scheme in $[7,8]$ ("GPF" for short ) and the proposed flexible feedback bits scheme ("FFB" for short) with $n_{T} p=4$ transmit antennas. For this case $p=2$, and the GPF scheme can only feedback 1 bit, whereas the proposed scheme can feedback more bits to improve the system performance. For comparison, in Figure 1 we also give the BER figures of the complex orthogonal code for four transmit antennas [11], and the numerical results of the upper bound in (24) of the proposed scheme. Figure 1 shows that with 1 bit feedback, the GPF and FFB schemes have close performance, whereas the FFB scheme has better performance with more feedback bits. In comparison to the complex orthogonal code, both two schemes have better performance.
In Figure 2, the BER performance of the two schemes with $n_{T} p=8$ transmit antennas is depicted. For this case $p=4$, and the GPF scheme can only feedback 3 bits, whereas the proposed FFB scheme can feedback more bits. We can observe that with the same feedback bits 3 , the two schemes have very similar performance, and with more feedback bits, the proposed FFB scheme can further improve the performance. In the simulations of these two schemes, the exhaustive search over all possible feedback vectors is used.

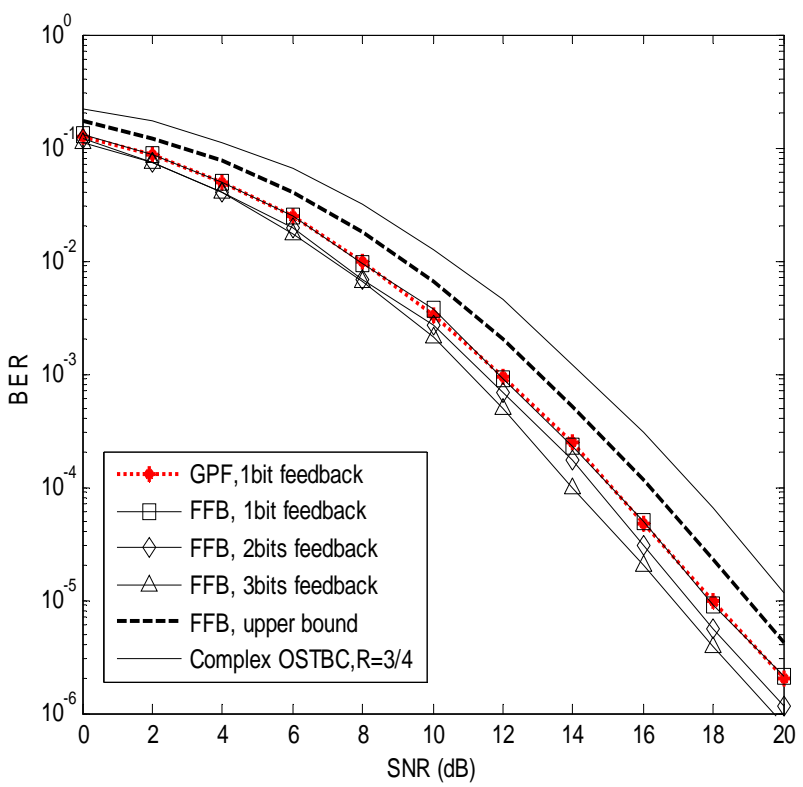

Figure 1. BER performance of the two schemes with $n_{T} p=4$ transmit antennas.

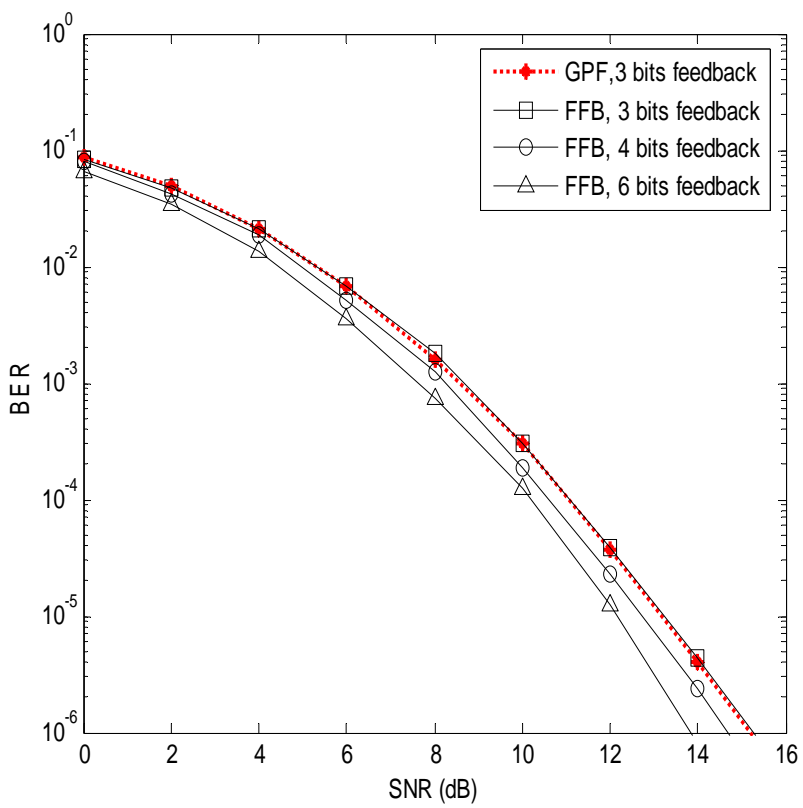

Figure 2. BER performance of the two schemes with $n_{T} p=8$ transmit antennas. 


\section{Conclusions}

In this paper, we proposed a partial feedback based OSTBC scheme with flexible feedback bits. The new scheme inherits the OSTBC properties of achieving full diversity, preserving low decoding complexity, and has full rate. Moreover, compared with the conventional partial feedback based OSTBC schemes, the new scheme can support flexible feedback bits and can improve the system performance with more feedback bits.

\section{REFERENCES}

[1] V. Tarokh, H. Jafarkhani and A. R. Calderbank, "Space-time Block Codes from Orthogonal Designs," IEEE Transactions on Information Theory, Vol. 45, No. 5, 1999, pp. 1456-1467.doi:10.1109/18.771146

[2] S. M. Alamouti, “A Simple Transmitter Diversity Scheme for Wireless Communications," IEEE Journal on Selected Areas in Communications, Vol. 16, No. 8, 1998, pp. 1451-1458.doi:10.1109/49.730453

[3] S. Sandhu and A. J. Paulraj, "Space-time Block Codes: A Capacity Perspective,” IEEE, Communications Letters, Vol. 4, No. 12, 2000, pp. 384-386. doi:10.1109/4234.898716

[4] H. Jafarkhani, “A Quasi-orthogonal Space-time Block Code," IEEE Transactions on Communications, Vol. 49, No. 1, 2001, pp. 1-4. doi:10.1109/26.898239
[5] W. Su and X. G. Xia, "Signal Constellations for Quasiorthogonal Space-time Block Codes with Full Diversity," IEEE Transactions on Information Theory, Vol. 50, 2004, pp. 2331-2347. doi:10.1109/TIT.2004.834740

[6] X. L. Ma and G. B. Giannakis, "Full-diversity Full-rate Complex-field Space-time Coding," IEEE Transactions on Signal Processing, Vol. 51, No. 11, 2003, pp. 2917-2930.doi:10.1109/TSP.2003.818206

[7] J. Akhtar and D. Gesbert, "Extending Orthogonal Block Codes with Partial Feedback," IEEE Transactions on Wireless Communications, Vol. 3, No. 6, 2004, pp. 1959-1962.doi:10.1109/TWC.2004.837469

[8] A. Sezgin, G. Altay and A. Paulraj, "Generalized Partial Feedback Based Orthogonal Space-time Block Coding," IEEE Transactions on Wireless Communications, Vol. 8, No. 6, 2009, pp. 2771-2775. doi:10.1109/TWC.2009.080352

[9] B. Hassibi and B. M. Hochwald, "High-rate Codes That Are Linear in Space and Time," IEEE Transactions on Information Theory, Vol. 48, No. 7, 2002, pp. 1804-1824. doi:10.1109/TIT.2002.1013127

[10] M. K. Simon and M. S. Alouini, "Digital Communication over Fading Channels,” John Wiley \& Sons Inc., 2000.

[11] G. Ganeon and P. Stoica, "Space-time Block Codes: A Maximum SNR Approach,” IEEE Transactions Informations Theory, Vol. 47, No. 4, 2001, pp. 1650-1656. doi:10.1109/18.923754 